\title{
The effect of surface relaxation and atomic vibration on the equilibrium shape of gold and copper crystallites
}

\author{
R. Najafabadia,* and D.J. Srolovitz ${ }^{\mathrm{a}, \mathrm{b}}$ \\ ${ }^{a}$ Department of Materials Science and Engineering, University of Michigan, Ann Arbor, MI 48109-2136, U.S.A. \\ ${ }^{b}$ Department of Materials and Interfaces, Weizmann Institute of Science, Rehovot 76100, Israel
}

Received 1 February 1994

Accepted 14 February 1994

Key words: Atomistic simulation; Wulff construction; Surface free energy

\section{SUMMARY}

The free energy of surfaces along the $<011>$ pole in gold and copper is determined to assess the effect of surface relaxation and atomic vibration on the equilibrium crystal shape of gold and copper. The Wulff construction is performed on the $\gamma$-plots to determine the equilibrium shape of gold and copper crystallites at different temperatures. It is shown that surface relaxation and atomic vibration do not have any discernible effect on the equilibrium shape of EAM gold or copper crystallites. The equilibrium shape of EAM gold crystallites is formed entirely from $\{111\}$ and $\{100\}$ facets, while that of EAM copper shows small $\{110\}$ facets in addition to the $\{111\}$ and $\{100\}$ facets.

\section{INTRODUCTION}

The free energy of a crystal surface is a function of its crystallographic orientation. This orientation dependence determines the equilibrium shape of crystallites [1,2]. The surface of an equilibrium crystallite may be determined by the Wulff construction, which is the Legendre transformation of the orientation-dependent surface free energy plot $(\gamma-$ plot $)$ [3]. The experimental determination of the equilibrium shape of crystals has been limited to a few cases [4-7] and it is generally carried out at elevated temperatures. Since the driving force for changes in crystal shape is relatively weak, in practice it is very difficult to achieve equilibrium crystallite shape, because of slow shape-change kinetics.

Theoretical calculations of the surface free energy have been performed with a simple lattice gas model, employing a terrace ledge kink approach [8] or a mean field approximation [9]. The results of these studies show that the equilibrium crystallite shape is completely faceted, with sharp edges at zero temperature and partially or completely rounded at elevated temperature. The

*To whom correspondence should be addressed. 
effect of surface relaxation and atomic vibration on the surface free energy is generally ignored. In a recent study on the determination of the silicon equilibrium shape near the (001) surface, the effect of (001) vicinal surface relaxation on the equilibrium shape of the silicon model was considered in terms of an elastic description of step-step interaction [10].

In this study, we examine the effect of surface relaxation and atomic vibration on the free energy of surfaces along the $<011>$ pole in gold and copper. In order to assess the importance of these effects on the equilibrium shape of a crystal, we do not consider the effect of thermal diffusion of steps (i.e., step wandering). It is well known [8,9] that such step wandering is one of the principal driving forces for the temporal evolution of the equilibrium shape at elevated temperature. We also do not consider the effect of surface reconstruction on the equilibrium shape. The embedded atom potentials (EAM) [11] are employed to represent the interatomic interactions in fcc gold and copper and the local harmonic model (LH) [12] is applied to determine the contribution of atomic vibration to the free energy.

\section{THEORY}

\section{Surface orientations}

Figure 1 shows a (011) stereographic projection of an fcc lattice. There is a twofold symmetry along the [011] axis and the $\{011\}$ mirror operation projects a plane onto another plane belonging to the same plane family. Thus, in order to determine the dependence of the surface free energy on the surface orientation, one only needs to consider the first octant of the stereographic sphere. The surfaces considered in the present study all contain the [011] direction and are projected onto the circle shown in Fig. 1. The crystallographic orientation of these surfaces is ( $m \bar{n} n)$, which contains the two directions $[0 \overline{1} 1]$ and $[2 \bar{n} \bar{m} \mathrm{~m}]$. We only consider surface planes with an angle $0 \leq \theta \leq 90$, where

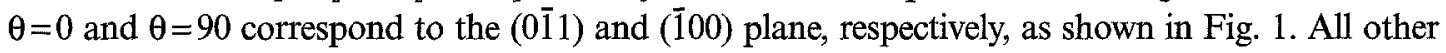
surfaces on the circle can be obtained from these surfaces by application of the symmetry elements.

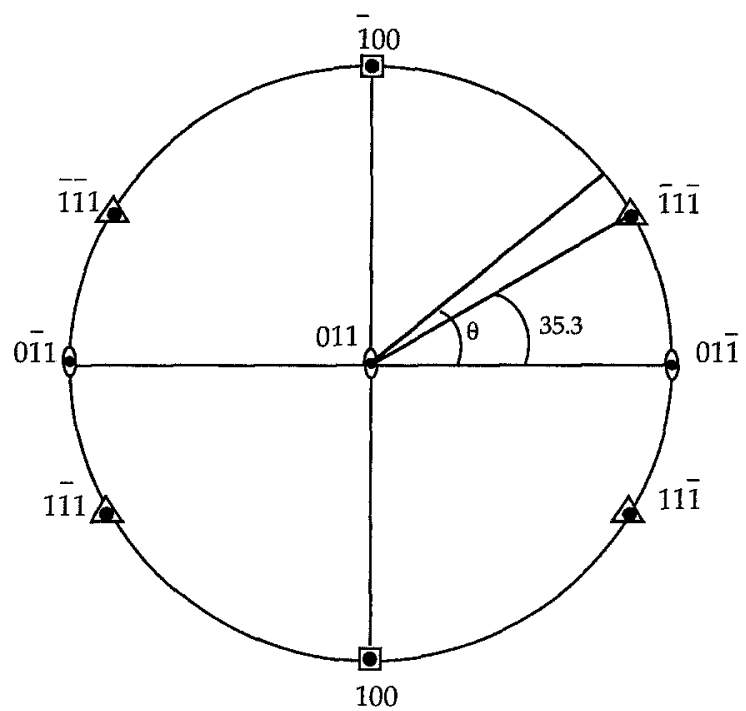

Fig. 1. The stereographic projection of an fcc lattice onto the (011) plane. All surfaces shown are parallel to the [011] pole. 
TABLE 1

THE CRYSTALLOGRAPHIC ORIENTATIONS OF THE SURFACES ALONG THE [011] POLE IN AN FCC LATTICE

\begin{tabular}{llllll}
\hline$(\mathrm{hkl})$ & $\theta$ (degrees) & $\mathrm{d}_{\text {hk }} / \mathrm{a}$ & $(\mathrm{hkl})$ & $\boldsymbol{\theta}$ (degrees) & $\mathrm{d}_{\text {hkl }} / \mathrm{a}$ \\
\hline$(\mathbf{0}-\mathbf{1 ~ 1 )}$ & $\mathbf{0 . 0 0}$ & $\mathbf{0 . 3 5 3 5 5 3}$ & $(5-44)$ & 41.47 & 0.066227 \\
$(1-1010)$ & 4.04 & 0.035267 & $(4-33)$ & 43.31 & 0.085749 \\
$(1-88)$ & 5.05 & 0.044023 & $(7-55)$ & 44.71 & 0.100504 \\
$(1-66)$ & 6.72 & 0.058521 & $(3-22)$ & 46.68 & 0.121268 \\
$(2-99)$ & 8.93 & 0.038808 & $(5-33)$ & 49.68 & 0.152499 \\
$(2-77)$ & 11.42 & 0.049507 & $(9-55)$ & 51.84 & 0.087370 \\
$(1-33)$ & 13.26 & 0.229416 & $(2-11)$ & 54.73 & 0.204124 \\
$(3-88)$ & 14.85 & 0.042718 & $(9-44)$ & 57.84 & 0.047036 \\
$(3-77)$ & 16.86 & 0.096674 & $(5-22)$ & 60.50 & 0.087039 \\
$(1-22)$ & 19.47 & 0.166667 & $(3-11)$ & $\mathbf{6 4 . 7 6}$ & $\mathbf{0 . 3 0 1 5 1 1}$ \\
$(4-77)$ & 22.00 & 0.046829 & $(10-33)$ & 67.01 & 0.046029 \\
$(5-88)$ & 23.84 & 0.040423 & $(7-22)$ & 67.99 & 0.066227 \\
$(2-33)$ & 25.24 & 0.106600 & $(4-11)$ & 70.52 & 0.117851 \\
$(5-77)$ & 26.79 & 0.090167 & $(9-22)$ & 72.55 & 0.053000 \\
$(7-99)$ & 28.80 & 0.068843 & $(5-11)$ & 74.20 & 0.192450 \\
$(5-66)$ & 30.50 & 0.050767 & $(6-11)$ & 76.73 & 0.081111 \\
$(7-88)$ & 31.74 & 0.037582 & $(7-11)$ & 78.57 & 0.140028 \\
$(9-1010)$ & 32.47 & 0.029827 & $(8-11)$ & 79.97 & 0.061546 \\
$(\mathbf{1 - 1 1})$ & 35.26 & $\mathbf{0 . 5 7 7 3 5 0}$ & $(9-11)$ & 81.06 & 0.109764 \\
$(10-99)$ & 38.15 & 0.030890 & $(10-11)$ & 81.95 & 0.049507 \\
$(8-77)$ & 38.94 & 0.039284 & $(\mathbf{1 0 0})$ & $\mathbf{9 0 . 0 0}$ & $\mathbf{0 . 5 0 0 0 0 0}$ \\
$(6-55)$ & 40.31 & 0.053916 & & & \\
\hline
\end{tabular}

All orientations shown have been simulated in this study. The table also contains the angles $\theta$ that the surfaces make with the $(0 \overline{1} 1)$ plane and their interplanar spacing $d_{h k l}$. The highlighted surfaces correspond to the surfaces with the densest planes.

Atomistic simulations were performed for all of the surfaces listed in Table 1. As indicated in the table, the four densest surface planes along the [011] pole are $(1 \overline{1} 1),(100),(0 \overline{1} 1)$ and $(3 \overline{1} 1)$.

\section{Energy of vicinal surfaces}

Any surface corresponding to a point on the circle (shown in Fig. 1) may be decomposed into terraces parallel to the [011] axis containing [01 $\overline{1}]$ steps. We only consider decompositions that involve low-index, high-symmetry terraces, such as the (001), (011) and (11) surfaces. In general, the energy of a vicinal surface may be written in terms of the energy of the corresponding flat surface and the energy associated with the [011] steps on the corresponding flat surface.

$$
\begin{aligned}
& \gamma_{\mathrm{v}}^{\mathrm{u}}=\gamma_{\mathrm{f}}^{\mathrm{u}} \cos (\phi)+\frac{\gamma_{\mathrm{s}}^{\mathrm{u}}}{\mathrm{h}} \sin (|\phi|) \\
& \gamma_{\mathrm{v}}^{\mathrm{r}}=\gamma_{\mathrm{f}}^{\mathrm{r}} \cos (\phi)+\frac{\gamma_{\mathrm{s}}^{\mathrm{r}}}{\mathrm{h}} \sin (|\phi|)+\gamma_{\text {int }} \\
& \Delta \gamma_{\mathrm{v}}=\Delta \gamma_{\mathrm{f}} \cos (\phi)+\frac{\Delta \gamma_{\mathrm{s}}}{\mathrm{h}} \sin (|\phi|)-\gamma_{\mathrm{int}}
\end{aligned}
$$


Here, $\gamma_{\mathrm{v}}, \gamma_{\mathrm{f}}$ and $\gamma_{\mathrm{s}}$ are the vicinal and flat surface energies and step energy, respectively, and the vicinal angle $\phi$ represents the degree of deviation from the flat surface orientation. The superscripts $\mathrm{u}$ and $\mathrm{r}$ stand for unrelaxed (bulk-terminated) and relaxed (traction-free) surface structure, respectively. $\Delta y$ is the relaxation energy and it is the difference between the energies of unrelaxed and relaxed structure. Parameter $h$ is the step height, which depends on the orientation of the flat surface. $\gamma_{\text {int }}$ is the total elastic interaction energy between steps; it is proportional to the square of the inverse step separation for small vicinal angle [13-15]. There is no step interaction energy in Eq. la, since the steps have no elastic fields on unrelaxed surfaces. The average step separation is simply $\mathrm{h} / \sin (\phi)$ and $\gamma_{\text {int }}$ is $\left(\gamma_{0} / \mathrm{h}^{2}\right) \sin ^{2}(\phi)$, where $\gamma_{0}$ depends on the step and flat surface orientations.

In the small angle $(\phi)$ limit, the vicinal surface energies may be expanded about $\phi=0$ :

$$
\begin{aligned}
& \gamma_{\mathrm{v}}^{\mathrm{u}}=\gamma_{\mathrm{f}}^{\mathrm{u}}+\frac{\gamma_{\mathrm{s}}^{\mathrm{u}}}{\mathrm{h}}|\phi|-\gamma_{\mathrm{f}}^{\mathrm{u}} \phi^{2} \\
& \gamma_{\mathrm{v}}^{\mathrm{r}}=\gamma_{\mathrm{f}}^{\mathrm{r}}+\frac{\gamma_{\mathrm{s}}^{\mathrm{r}}}{\mathrm{h}}|\phi|-\left(\gamma_{\mathrm{f}}^{\mathrm{r}}-\frac{\gamma_{0}}{\mathrm{~h}^{2}}\right) \phi^{2} \\
& \Delta \gamma_{\mathrm{v}}=\Delta \gamma_{\mathrm{f}}+\frac{\Delta \gamma_{\mathrm{s}}}{\mathrm{h}}|\phi|-\left(\Delta \gamma_{\mathrm{f}}-\frac{\gamma_{0}}{\mathrm{~h}^{2}}\right) \phi^{2}
\end{aligned}
$$

where we have neglected terms higher than second order in $\phi$. These relationships may be employed to identify the cusps on the $\gamma$-plot and to determine the steps free energies.

\section{Free energy minimization method}

The Helmholtz free energy of a single-component solid consists of two energy contributions, i.e., the static (bonding) energy and the energy associated with atomic vibrations. The latter is an explicit function of temperature and classically vanishes at zero temperature.

$$
F(T, V, N)=E_{o}\left(r_{1}, \ldots, r_{N} ; V\right)+E_{v i b}\left(r_{1}, \ldots, r_{N} ; T, V\right)
$$

The static energy, $\mathrm{E}_{\mathrm{o}}$, is determined employing the EAM potentials [11]. At finite temperature, the vibrational contribution to the free energy is determined within the framework of the Local Harmonic model [12]. In this model, each atom is assumed to vibrate within a potential field which may be characterized by three independent frequencies. These frequencies are determined in terms of the local dynamical matrix of each atom $D_{i \alpha, \beta}=\partial^{2} E_{o} /\left(\partial r_{i \alpha} \partial r_{i \beta}\right)$, where $r_{i \alpha}$ is the $\alpha$ coordinate of atom $i$. The classical vibrational contribution to the free energy within this model is

$$
E_{v i b}=3 k_{B} T \sum_{i} \ln \left(\frac{h\left|D_{i}\right|^{1 / 6}}{m^{1 / 2} k_{B} T}\right)
$$

The surface free energy, $\gamma$, is the difference between the free energy of a system containing a surface and that of a perfect crystal with the same number of atoms; it is generally normalized by the surface area. 


\section{RESULTS AND DISCUSSION}

In order to determine the free energies of the (mn̄n) surfaces of Table 1 , the free energy expression of Eq. 3 was minimized (using the conjugate gradient method) with respect to the position of all atoms. Parallel to the surface plane, periodic boundary conditions are applied (i.e., in both the $[2 \overline{\mathbf{n}} \overline{\mathrm{m}} \mathrm{m}]$ and $[01 \overline{1}]$ directions). Normal to the surface plane (z-direction), however, the atoms which are free to move are attached to a perfect (unstrained) fcc crystal with the appropriate lattice parameter. During the course of the free energy minimization, the size of the computational cell in the $\mathrm{z}$-direction was increased until the surface free energy remained unchanged $\left(<10^{-9} \mathrm{~J} / \mathrm{m}^{2}\right)$ upon further extension and the forces on the atoms were less than $10^{-5} \mathrm{eV} / \AA$. We note that, due to the long-range strain field associated with high-index surfaces, the size of the simulation cell in the $z$-direction was not the same for all surfaces. For example, as shown in Fig. 2, the normal interplanar strain $\left(\varepsilon_{z z}\right)$ associated with the $(0 \overline{1} 1)$ surface vanishes at approximately $1.5 \mathrm{a}_{\mathrm{o}}$ below the $(0 \overline{1} 1)$ surface, while at this distance the interplanar strain associated with the $(2 \overline{7} 7)$ surface is as large as that on the top layer in the (011) surface and is significant until approximately $4 \mathrm{a}_{\mathrm{o}}$ below the $(2 \overline{7} 7)$ surface.

\section{Zero temperature}

Figure 3 shows the gold and copper zero temperature surface free energies as a function of the angle $\theta$ for all of the surfaces listed in Table 1. The surface relaxation energy (i.e., the difference between the surface free energy of the unrelaxed and relaxed surfaces) is about 6 to $10 \%$ of the total surface free energy for the gold surfaces, while it is less than $1 \%$ for the copper surfaces. Thus, the surface-induced strain field contributes much more to the surface energies in gold than in copper (the elastic constants of copper and gold are similar). Figure 3 clearly shows cusps in the surface energy versus surface orientation for both gold and copper at $\theta=35.26^{\circ}$ and $90^{\circ}$, corresponding to the (111) and (100) flat surfaces, respectively. The maximum surface energy occurs at $\theta=64.76^{\circ}$, corresponding to the (3i1) surface. It is difficult to determine whether the slope of the surface energy versus orientation, $\partial \gamma_{\mathrm{r}} / \partial \theta$, is continuous or not, due to the lack of
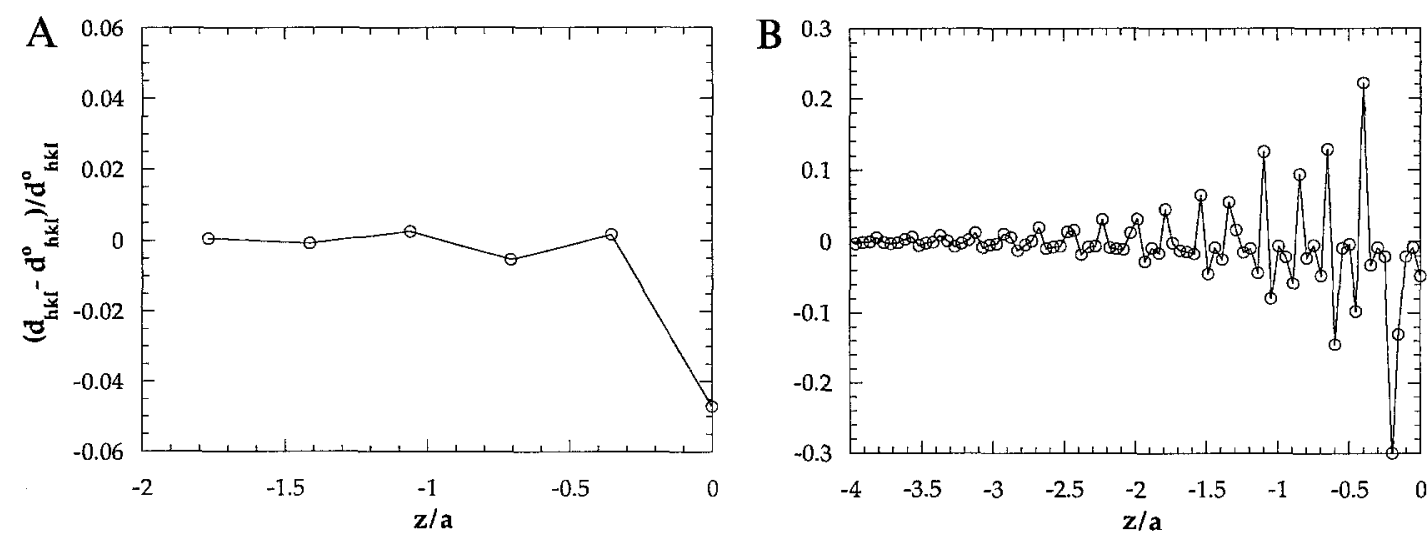

Fig. 2. The variation of the planar strain with the distance into the bulk (A) from the (0i1) surface and (B) from the $(2 \overline{7} 7)$ surface. $d^{0}$ is the bulk (hkl) planar spacing and $d$ is the spacing between two immediately adjacent (hkl) planes of the (hkl) surface. 

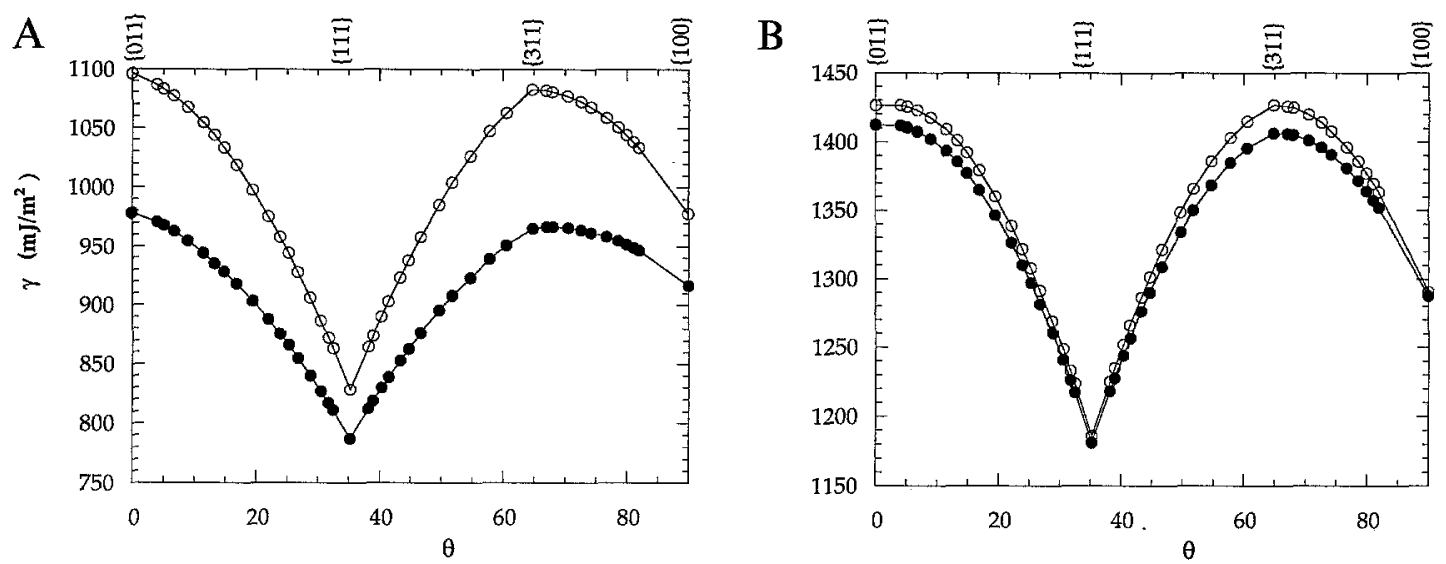

Fig. 3. The variation of the $(m \bar{n} n)$ surface energy $(T=0)$ with surface orientation $\theta$ for EAM (A) gold and (B) copper. The open and filled circles represent the unrelaxed and relaxed surface energies, respectively.

data points close to $64.76^{\circ}$ in Fig. 3 (see especially the maximum at the (3ī1) surface orientation of gold). An additional maximum occurs at $\theta=0$, which corresponds to the flat $(0 \overline{1} 1)$ surface, because of the $\{011\}$ mirror symmetry.

\section{Finite temperature}

The free energy minimization method, described in the previous section, and the EAM potentials were employed to determine the surface free energies of gold and copper at several different temperatures. Figure 4 shows how, in the classical limit, the free energies of the (100), (011), (311) and (722) surfaces in gold and copper vary with temperature. The surface free energies exhibit a nearly linear decrease with increasing temperature. This suggests that the surfaces have positive excess entropies. Figure 4 shows that the slopes of the free energy curves for copper are approximately twice as large as those for gold. Thus, copper surfaces typically have higher (circa two times) entropies than the corresponding gold surfaces. It was recently shown [17] that, while the
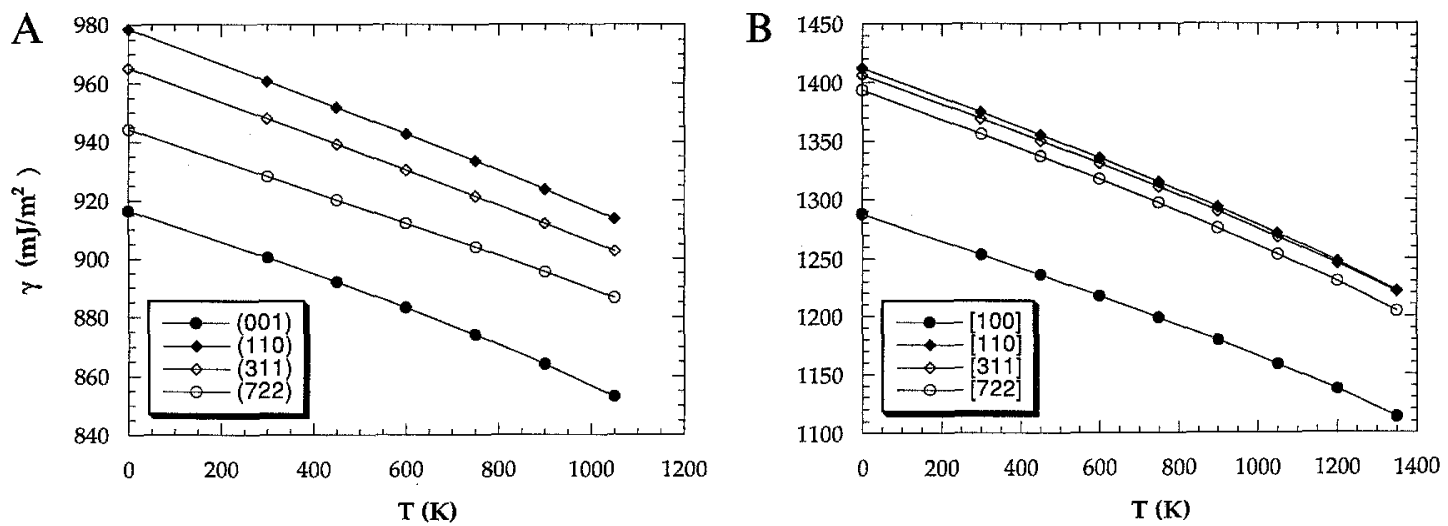

Fig. 4. The variation of the $(m \bar{n} n)$ surface free energy with temperature for several (A) gold and (B) copper surfaces. 

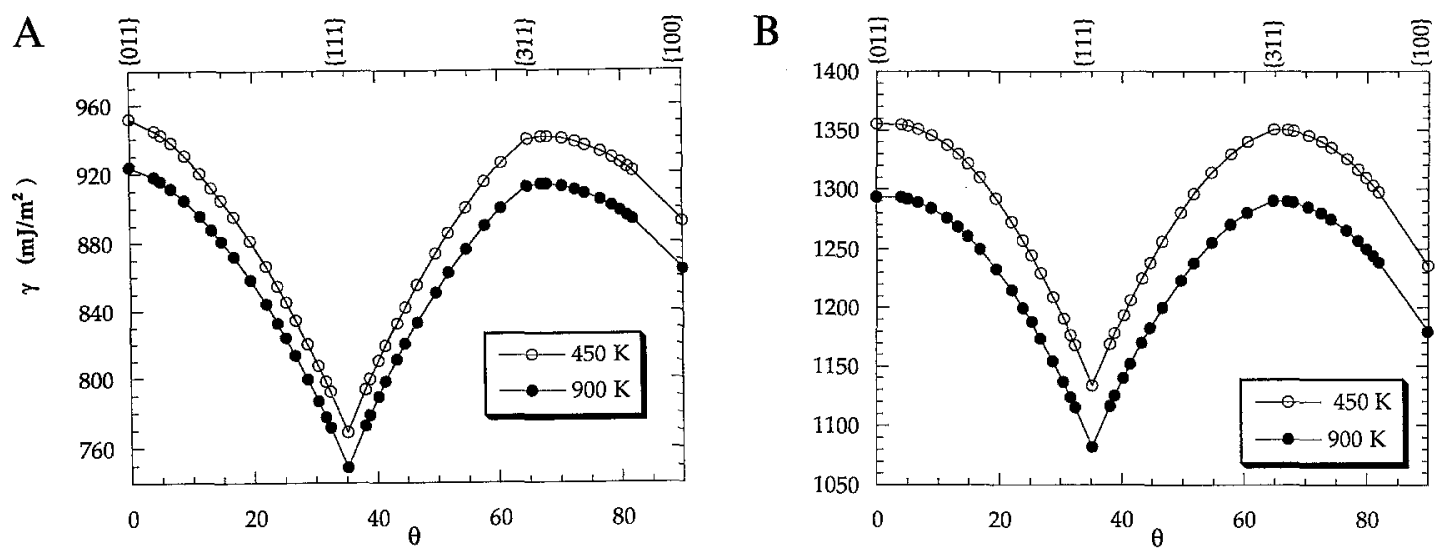

Fig. 5. The variation of the (mn̄n) surface free energy with surface orientation $\theta$ at $T=450$ and $900 \mathrm{~K}$ for (A) gold and (B) copper.

free energies of perfect crystals determined by the free energy minimization method employing the LH model and the EAM potential are in good agreement with Monte Carlo/thermodynamic integration results, at high temperatures, defect entropies determined by the Monte Carlo/thermodynamic integration method show a stronger dependence on temperature than found using the LH model. This deviation can be attributed largely to anharmonicity.

Figure 5 shows the variation of the surface free energy in gold and copper with angle $\theta$ for 450 and $900 \mathrm{~K}$. The surface free energy curves for these two temperatures are very similar and also resemble the free energy curves at zero temperature (see Fig. 3). Moreover, the locations of the energy cusps and maxima on the free energy vs. orientation curves have remained the same as those on the zero-temperature free energy curves. This suggests that these features are determined primarily by the fcc crystal structure of these materials.

The surface enthalpy (h) and entropy (s) of the gold and copper surfaces were obtained by
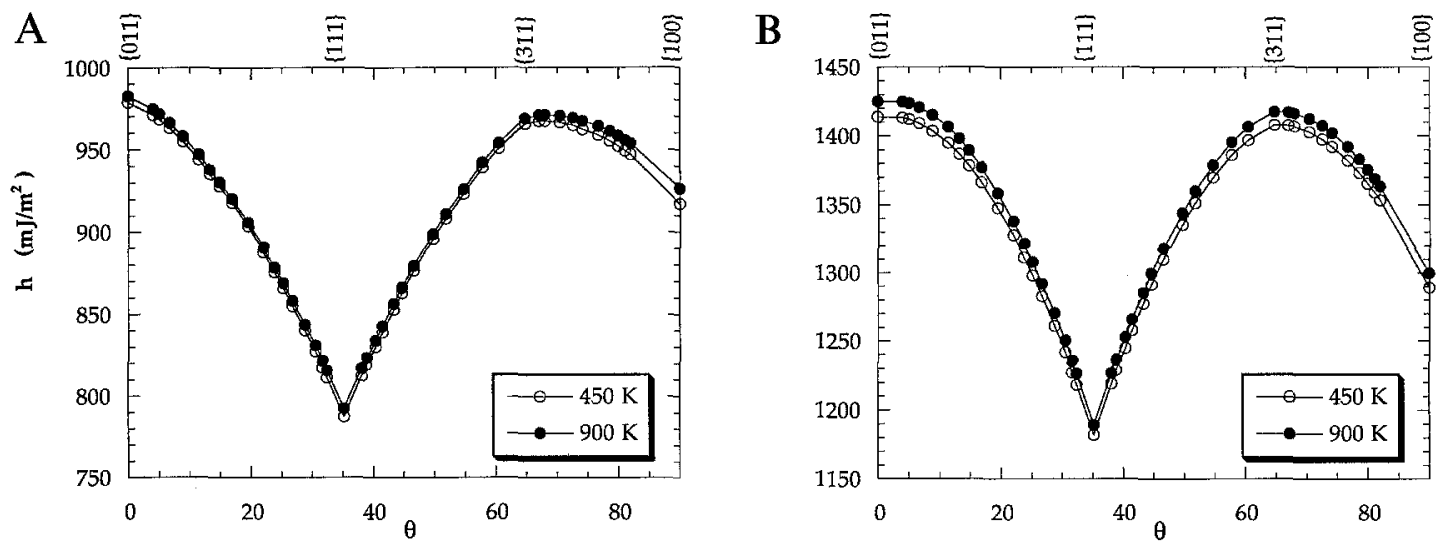

Fig. 6. The variation of the ( $\mathrm{mnn}$ ) surface enthalpy with surface orientation $\theta$ at $\mathrm{T}=450$ and $900 \mathrm{~K}$ for $(\mathrm{A})$ gold and (B) copper. 

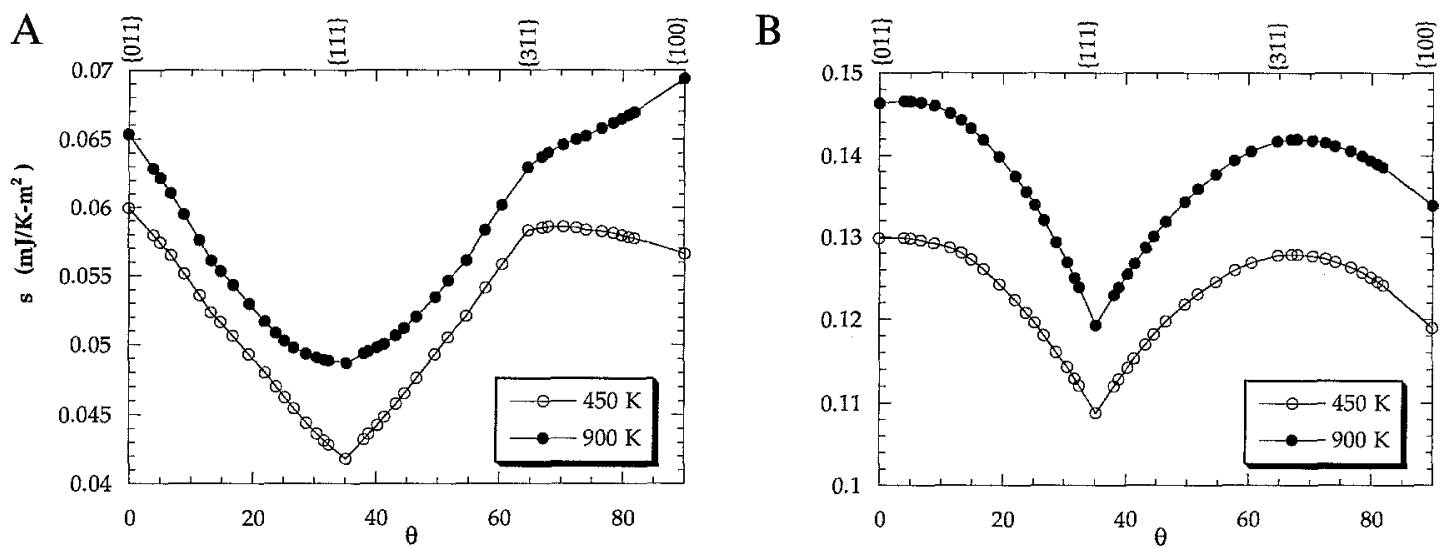

Fig. 7. The variation of the (mn̄n) surface entropy with surface orientation $\theta$ at $T=450$ and $900 \mathrm{~K}$ for (A) gold and (B) copper.

fitting a third-order polynomial function to the surface free energy data and making use of the simple thermodynamic relationships $s=\partial \gamma(\mathrm{T}) / \partial \mathrm{T}$ and $\mathrm{h}=\gamma-\mathrm{sT}$. Figure 6 shows how the gold and copper surface enthalpies vary with angle $\theta$ at 450 and $900 \mathrm{~K}$. The surface enthalpy increases with increasing temperature and the rate of the enthalpy increase (surface specific heat) in copper is larger than that in gold.

The surface entropies at 450 and $900 \mathrm{~K}$ are shown in Fig. 7. The general trends are the same as for the free energy curves. However, a marked difference occurs for the large $\theta$ surfaces in gold at $900 \mathrm{~K}$ (the EAM gold melting temperature is $1050 \mathrm{~K}$ [16]). Here, the surface excess entropy increases with increasing angle for orientations above $64.76^{\circ}$, while it decreases with increasing angle at $450 \mathrm{~K}$ in gold and both at 450 and $900 \mathrm{~K}$ in copper. At $900 \mathrm{~K}$, the entropy of the (100) gold surface is larger than for any other gold surface at that temperature, i.e., approximately $7.10^{-5} \mathrm{~J} / \mathrm{K} \mathrm{m}^{2}$. At both 450 and $900 \mathrm{~K}$, the gold surface excess entropy shows cusps at $64.76^{\circ}$ (i.e., the (311) surface orientation), while no such discontinuities in the slope $\partial \mathrm{s} / \partial \theta$ occur in copper. Furthermore, the strong cusp in the surface excess entropy observed in gold at $450 \mathrm{~K}$ and in copper at 450 and $900 \mathrm{~K}$ (i.e., at the $\{111\}$ orientation), has been replaced with a smooth minimum in gold at $450 \mathrm{~K}$. At $\theta=0$, corresponding to the $(0 \overline{1} 1)$ surface, the surface entropy curves for gold at 450 and $900 \mathrm{~K}$ show an inverse cusp, while no cusp was detected for this surface orientation on the copper surface entropy curves.

\section{Wulff construction}

The equilibrium shape of the EAM gold and copper crystallites may be determined from their corresponding surface free energy curves by means of the Wulff construction [2]. Alternatively, if the surface free energy curve can be expressed in terms of the surface orientations in an analytical form, one may use the Legendre transformation [3] to determine the functional form of the equilibrium shape, $z(x, y)$. In the present study, we determined the equilibrium shape by employing the Wulff construction method (partly because only a finite number of surface energy data points is available here). In this method, the surface energy is plotted versus surface orientation in a polar representation, $\mathrm{R}(\mathrm{r}, \theta)=\mathrm{R}\left(\gamma_{\mathrm{r}}, \phi\right)$. A plane, perpendicular to the radius vector, is drawn at a 
distance proportional to $\mathrm{R}$ for each point on the surface free energy curve. The volume enveloped by these planes is the equilibrium shape of the crystallite. This shape minimizes the free energy of the crystallite for fixed volume. Figure 8 shows the equilibrium shape of the EAM gold and copper crystallites, determined from the relaxed surface free energy at zero temperature (see Fig. 3 ). The twofold symmetries along the $\{011\}$ axis and $\{011\}$ mirror plane are clearly seen in these equilibrium shapes. The equilibrium shape of EAM gold consists entirely of $\{111\}$ and $\{100\}$ facets, while the equilibrium shape of EAM copper has small $\{110\}$ facets as well as $\{111\}$ and $\{100\}$ facets at zero temperature.

The equilibrium shapes of gold and copper crystallites, determined using the unrelaxed surface free energies, consist of the same facets as those seen in Fig. 8, with the proportion of the facets on the equilibrium shapes essentially unchanged. This shows that, while surface relaxation reduces the free energy of gold and copper surfaces by up to 10 and $1 \%$, respectively, relaxation has no significant effect on the anisotropy of the surface free energy or the equilibrium shape of EAM gold and copper crystallites.

We also determined the equilibrium shape of gold and copper at several temperatures, based on the surface free energies presented in the section on finite temperature. Again, we found that the equilibrium crystallite shapes were very similar to those determined at zero temperature. This suggests that surface vibration does not significantly affect the surface free energy anisotropy or the equilibrium shape of EAM gold or copper crystallites.

The equilibrium shape of EAM copper shows the presence of $\{110\}$ facets, while these facets are absent in EAM gold (see Fig. 8). The equilibrium shapes of both gold and copper crystallites exhibit $\{111\}$ and $\{100\}$ facets. The formation of facets is associated with the presence of cusps on the surface free energy vs. orientation polar plot [2]. Equation 2 suggests that the slopes of the free energy vs. orientation curves $(\partial \gamma / \partial \phi)$ on the two sides of a cusp are of opposite sign and
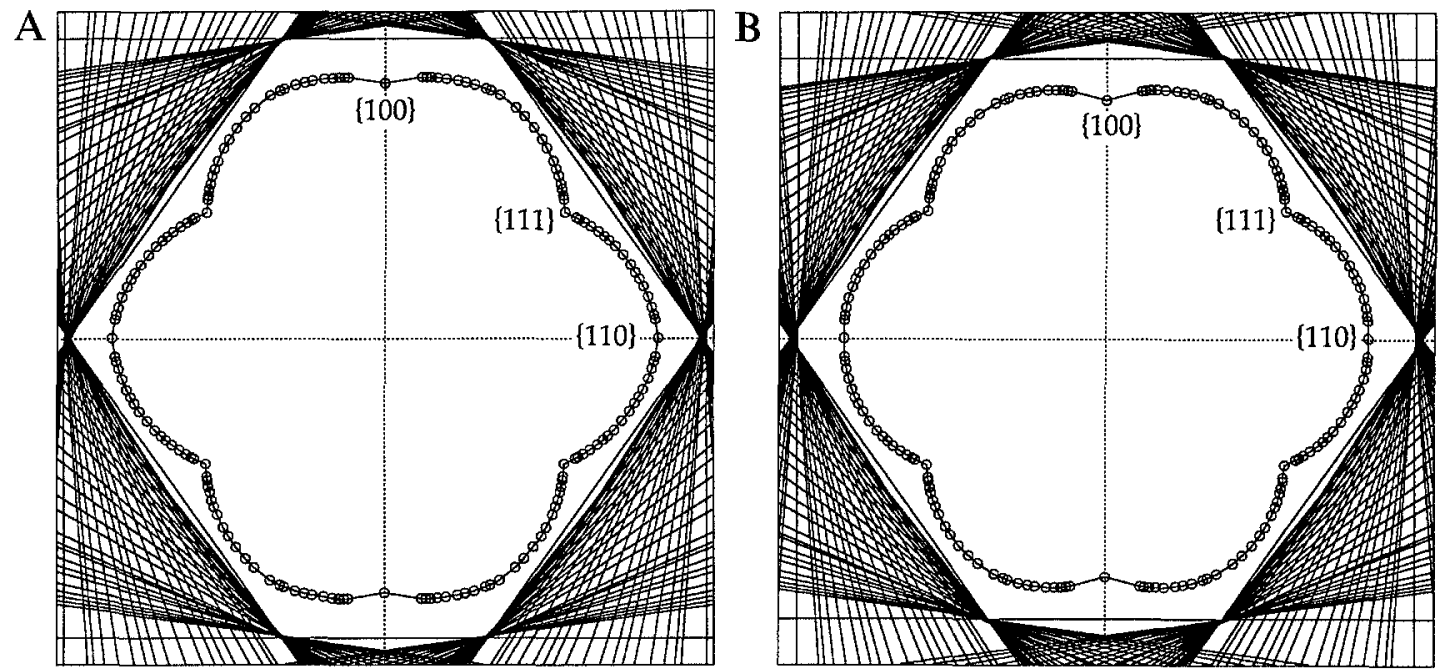

Fig. 8. The Wulff construction of the equilibrium shape, based on the relaxed surface free energy calculations at $T=0$ for (A) gold and (B) copper. The straight lines represent the projection of planes tangent to the $\gamma$-plot on the (011) plane. The circles represent the surface free energy in polar coordinates $(\gamma$-plot) and are scaled by 0.8 for clarity. 

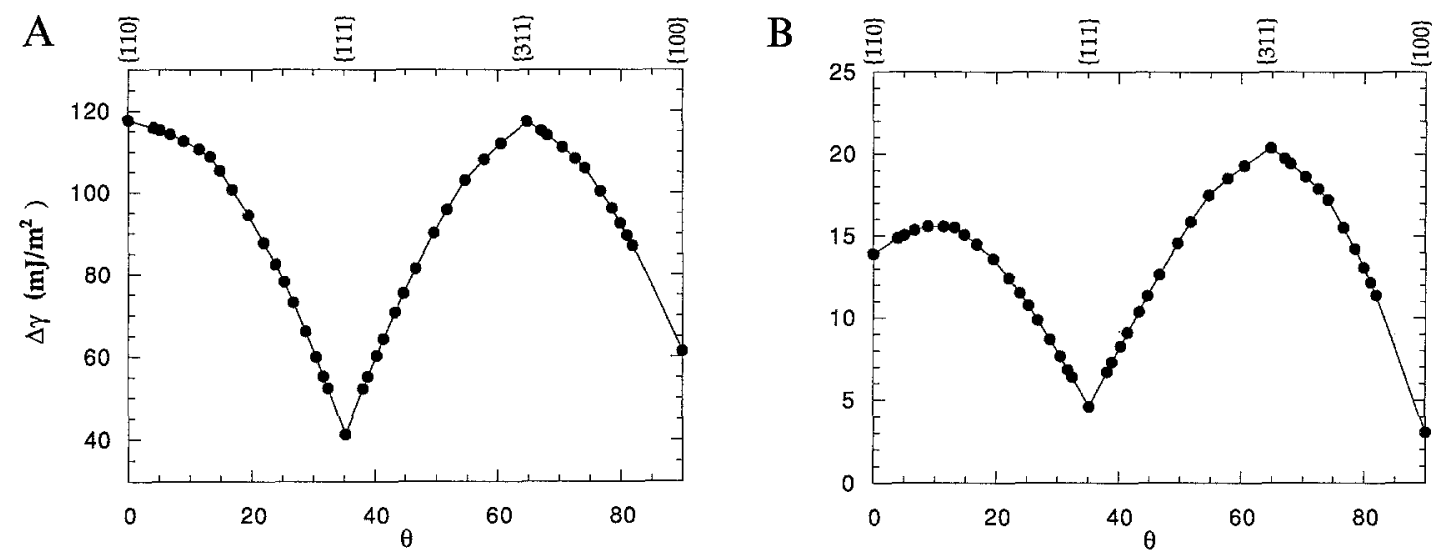

Fig. 9. The variation of the (mn̄n) surface relaxation energy with surface orientation $\theta$ at $T=0$ for (A) gold and (B) copper.

that the surface free energy should be linear within a small angular range around the cusp orientation $\theta_{0} . \alpha$, the ratio of the coefficients of the $\phi$ and $\phi^{2}$ terms in Eq. 2, determines the angular range over which this linear relation is valid. The surface relaxation energy as defined in Eq. $2 \mathrm{c}$ should show the same behavior around a cusp as the surface energy itself. Figure 9 shows how the surface relaxation energy varies with angle $\theta$ for both gold and copper. From this figure, it is easy to identify the cusps at the $(1 \overline{1} 1)$ and (100) surface orientations and an inverse cusp at the (3i1) surface on both surface relaxation energy curves (the intersection point between two straight line segments with slopes of opposite sign defines a cusp's position). At the (011) orientation on the copper surface relaxation energy curve, a cusp is observed, while the gold surface curve shows an inverse cusp. This is in agreement with the equilibrium shape results, which show $\{110\}$ facets on copper crystallites but not on gold crystallites.

It is possible that $\{011\}$ cusps, rather than inverse cusps, also exist for gold but could not be observed because of the discreteness of data points near the $\{011\}$ orientation. Furthermore, if the $\alpha$ ratio is very small for the near $\{011\}$ orientations, then cusps will also not be observable. Finally, we note that, although reconstruction was not allowed in the present study, the gold $\{011\}$ surface is known to undergo a $(1 \times 2)$ surface reconstruction. Potentially, this could lower the free energy of $\{011\}$ gold surfaces sufficiently for cusps to form.

The surface relaxation and free energy curves show a slight asymmetry around the (111) cusp (see Figs. 3 and 9) and, therefore, there is a small difference in the slopes of the energy on the positive and negative side of the ( $1 \overline{1} 1)$ cusp. The slope of the energy is proportional to the step energy (see Eq. 2) and, therefore, two kinds of [011] steps can be formed on the (111) flat surface. These are ascending (lower terrace A plane and upper terrace B plane) and descending (upper terrace A plane and lower terrace $C$ plane) steps. A similar asymmetry is also seen around the (3i1) inverse cusps.

\section{CONCLUSIONS}

We have determined the free energy of surfaces along the $<011>$ pole in gold and copper by employing EAM potentials and an approximate free energy, based on the LH model. The Wulff 
construction was performed on the $\gamma$-plots to determine the equilibrium shapes of EAM gold and copper for different temperatures. The equilibrium shape of gold along the $<011>$ pole consists entirely of $\{111\}$ and $\{100\}$ facets, while the equilibrium shape of copper shows small $\{110\}$ facets in addition to $\{111\}$ and $\{100\}$ facets. It was shown that, while both surface relaxation and atomic vibration reduce the surface free energy, they do not have any discernible effect on the equilibrium shape of EAM gold and copper crystallites, since the anisotropy of the surface free energy remains essentially unchanged.

\section{ACKNOWLEDGEMENTS}

D.J.S. gratefully acknowledges the hospitality of The Weizmann Institute of Science and the support of the Michael Visiting Professorship. This research was supported by the Division of Materials Science of the Office of Basic Energy Sciences of the U.S. Department of Energy, Grant No. FG02-88ER-45367.

\section{REFERENCES}

1 Wulff, G., Z. Krist. Mineral., 34 (1901) 449.

2 Herring, C., Phys. Rev., 82 (1951) 87.

3 Landau, L. and Lifshitz, L., Statistical Physics, 3rd ed., Part 1, Pergamon Press, Oxford, 1980, p. 521.

4 Heyraud, J.C. and Metois, J.J., Acta Metall., 28 (1980) 1789.

5 Heyraud, J.C. and Metois, J.J., Surf. Sci., 128 (1983) 334.

6 Metois, J.J. and Heyraud, J.C., Surf. Sci., 180 (1987) 647.

7 Eaglesham, D.J., White, A.E., Feldman, L.C., Moriya, N. and Jacobson, D.C., Phys. Rev. Lett., 70 (1993) 1643.

8 Gruber, E.E. and Mullins, W.W., J. Phys. Chem. Solids, 28 (1966) 875.

9 Rottman, C. and Wortis, M., Phys. Rev., B29 (1984) 328.

10 Tersoff, J. and Pehlke, E., Phys. Rev., B47 (1993) 4072.

11 Foiles, S.M., Baskes, M.I. and Daw, M.S., Phys. Rev., B33 (1986) 7983.

12 LeSar, R., Najafabadi, R. and Srolovitz, D.J., Phys. Rev. Lett., 63 (1989) 624.

13 Marchenko, V.I. and Parshin, A.Y., Sov. Phys. J. Exp. Theor. Phys., 52 (1981) 129.

14 Wolf, D. and Jaszczak, J.A., Surf. Sci., 277 (1992) 301.

15 Najafabadi, R. and Srolovitz, D.J., Surf. Sci., submitted for publication.

16 Foiles, S.M. and Adams, J.B., Phys. Rev., B40 (1989) 5909.

17 Foiles, S.M., Phys. Rev. B, (1994) in press. 